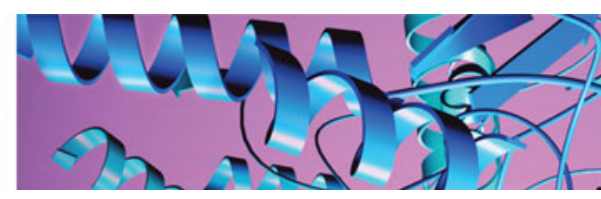

\title{
REPORT
}

\section{A stretched conformation of DNA with a biological role?}

\author{
Niklas Bosaeus ${ }^{1}$, Anna Reymer ${ }^{2}$, Tamás Beke-Somfai ${ }^{3}$, Tom Brown ${ }^{4}$, Masayuki Takahashi ${ }^{5}$, \\ Pernilla Wittung-Stafshede ${ }^{1}$, Sandra Rocha ${ }^{1}$ and Bengt Nordén ${ }^{6 *}$ \\ ${ }^{1}$ Biology and Biological Engineering, Chalmers University of Technology, Gothenburg, Sweden \\ ${ }^{2}$ Department of Chemistry and Molecular Biology, Gothenburg University, Gothenburg, Sweden \\ ${ }^{3}$ Institute of Materials and Environmental Chemistry, Hungarian Academy of Sciences, Budapest, Hungary \\ ${ }^{4}$ Department of Chemistry, University of Oxford, Oxford, U.K. \\ ${ }^{5}$ School of Bioscience and Biotechnology, Tokyo Institute of Technology, Tokyo, Japan \\ ${ }^{6}$ Chemistry and Chemical Engineering, Chalmers University of Technology, Gothenburg, Sweden
}

Quarterly Reviews of Biophysics (2017), 50, e11, page 1 of 11 doi:10.1017/S0033583517000099

Abstract. We have discovered a well-defined extended conformation of double-stranded DNA, which we call $\Sigma$-DNA, using laser-tweezers force-spectroscopy experiments. At a transition force corresponding to free energy change $\Delta G=1.57 \pm 0.12 \mathrm{kcal}\left(\mathrm{mol}\right.$ base pair) ${ }^{-1} 60$ or 122 base-pair long synthetic GC-rich sequences, when pulled by the $3^{\prime}-3^{\prime}$ strands, undergo a sharp transition to the $1.52 \pm 0.04$ times longer $\Sigma$-DNA. Intriguingly, the same degree of extension is also found in DNA complexes with recombinase proteins, such as bacterial RecA and eukaryotic Rad51. Despite vital importance to all biological organisms for survival, genome maintenance and evolution, the recombination reaction is not yet understood at atomic level. We here propose that the structural distortion represented by $\Sigma$-DNA, which is thus physically inherent to the nucleic acid, is related to how recombination proteins mediate recognition of sequence homology and execute strand exchange. Our hypothesis is that a homogeneously stretched DNA undergoes a 'disproportionation' into an inhomogeneous $\Sigma$-form consisting of triplets of locally $B$-like perpendicularly stacked bases. This structure may ensure improved fidelity of base-pair recognition and promote rejection in case of mismatch during homologous recombination reaction. Because a triplet is the length of a gene codon, we speculate that the structural physics of nucleic acids may have biased the evolution of recombinase proteins to exploit triplet base stacks and also the genetic code.

\section{Introduction}

Double-stranded (ds) DNA can adopt multiple conformations, exhibiting 'polymorphism', directly related to the physical properties of the molecule and to its biological function. The most well-known forms of dsDNA are the $B$-, $A$ - and $Z$-forms. The $B$-form is the 'normal' DNA found in most biological aqueous contexts. Under reduced water conditions the $A$-form is favoured, and under certain ionic and base sequence conditions the inverted $Z$-form prevails. Biological roles of both $A$ - and $Z$-DNA are possible (Arnott et al. 1986; Brown et al. 2000; Lu et al. 2000; Rich et al. 1984). Compared with many other polymers, DNA has a spectacular compactness and high stiffness ('persistence length') and with a high negative charge density

* Author for correspondence: B. Nordén, Chemistry and Chemical Engineering, Chalmers University of Technology. Email: norden@chalmers.se owing to the presence of close phosphate groups in the backbone. The high persistence length is in part due to the electrostatic repulsion of these moieties, partly to the local steric interactions of the coin pile-stacked nucleobases. The great stability of $B$-form DNA, needed to securely store the genomic information, is due to mainly the stacking of the bases, which in turn is due to hydrophobic effects (requiring surrounding bulk water) and to anisotropic dispersive forces (Friedman \& Honig, 1995). The base-pair hydrogen bonds, which are important for many biological processes, play only a partial role in the stability of DNA (Guckian et al. 2000) but are strengthened by the hydrophobic environment in the core of the close-packed bases (see below). DNA can be extended, twisted or unwound during diverse biological processes, such as DNA repair, chromatin compaction and gene regulation. Proteins that interact with 
DNA are able to exploit the unique structural properties of DNA, although it is not clear to what extent the polymorphic phase map is fully exploited. Taking the particular case of homologous recombination, the recombinase proteins RecA and Rad51 organize themselves in a helical manner around DNA, which is unwound and stretched to about $50 \%$ in length compared with the ds $B$-form (dsDNA). RecA and Rad51 play crucial roles in chaperoning homologous recombination and DNA repair by catalyzing strand exchange in, respectively, prokaryotes and eukaryotes. They use similar molecular reaction mechanisms for the strand exchange reaction and bind first to a single-stranded (ss) part of DNA with high cooperativity, in the presence of ATP, to form a filamentous complex in which DNA adopts an extended conformation (Flory et al. 1984). The ssDNARecA filament then interacts with an incoming dsDNA to form an ssDNA-RecA-dsDNA complex (Kiianitsa \& Stasiak, 1997) and, if the two DNAs have the same or similar sequence, strand exchange occurs.

Twenty-five years ago, we studied shear-aligned fibres of Escherischia coli RecA complexes with both ss as well as dsDNA in aqueous solution by small-angle neutron scattering (SANS) and UV linear dichroism (LD) spectroscopy under identical flow conditions (Nordén et al. 1992). The orientation created a well-resolved SANS pattern, where the helical diffraction cross provided exact information about the helix pitch and, most importantly, yielded the flow orientation distribution, making it possible to translate the LD signals into average base-plane orientations (Hagmar et al. 1992; Nordén et al. 1992). Electron microscopy had revealed that the DNA was extended by approximately a factor of 1.5 (Stasiak \& Di Capua, 1982; Stasiak et al. 1981), but surprisingly the base orientation concluded from LD did not show any inclination of the base planes (neither for ss- nor for dsDNA) as would be expected for a continuously stretched and unwound DNA form (Hagmar et al. 1992; Nordén et al. 1992, 1998). This observation remained puzzling until 2002, when the application of systematically mutated aromatic residues in RecA allowed a threedimensional model to be constructed for the aqueous solution structures of RecA-dsDNA and RecA-ssDNA (Morimatsu et al. 2002). From LD results combined with crystal data for RecA (Story et al. 1992), a model emerged in which the DNA was accommodated in an ordered way inside a helical arrangement of RecA monomers allowing the bases to be perpendicular (Morimatsu et al. 2002). A later crystal structure of RecA-dsDNA and RecA-ssDNA confirmed our conclusion: a near perpendicular nucleobase orientation and clustering of triplets of bases stacked approximately as in $B$-form DNA (Chen et al. 2008) (Fig. 1). Using LD spectroscopy together with site-specific tyrosine mutations, we found a similar nucleobase organization, perpendicular to the fibre axis of the complex, for the dsDNA complex with Rad51 in solution (Reymer et al. 2009). For the Rad51 complex with
ssDNA, however, a perpendicular base orientation was observed only in the presence of activating factors, such as $\mathrm{Ca}^{2+}$ ion or Swi5/Sfr1 protein (Fornander et al. 2014). Recent cryo-EM high-resolution structural analyses of activated human Rad51 in complex with DNA have demonstrated conserved features with the RecA system (Xu et al. 2017).

In 2012, we identified using single-molecule force spectroscopy on short synthetic DNAs, in the absence of recombination proteins, the existence of an overstretched state of DNA, which we shall here return to and study in detail. All evidence indicates it is a thermodynamically welldefined conformation. We will call it $\Sigma$-DNA, and it consists of a ca $50 \%$ extended stable conformation of ds (basepaired) GC-rich DNA, at a transition force of ca $64 \mathrm{pN}$ applied to the $3^{\prime}-3^{\prime}$ strands (Bosaeus et al. 2012). The $\Sigma$-form may be considered a special case of the wider group of stretched DNA forms that have been called 'S-DNA' some of which, though, appear less well defined. In contrast to the $\Sigma$-form observed for base-paired GC-rich DNA stretched along the $3^{\prime}-3^{\prime}$ ends, $S$-DNA is usually observed as a 70\% elongated form when stretching a long plasmid or phage DNA (Cluzel et al. 1996; Williams et al. 2002) and, at least in AT-rich DNA, S-DNA appears to involve denaturation (Bosaeus et al. 2012, 2014). The fact that the $\Sigma$-form has so long escaped discovery is thought to be due to that it is first recently stretch experiments on short synthetic DNA have been possible with high accuracy (Bosaeus et al. 2012, 2014).

The degree of extension that we observe for $\Sigma$-DNA, compared with $B$-DNA, is within experimental error the same as that found in complexes with the recombinase enzymes RecA and Rad51. One may thus ask if this is just a coincidence or if this structural distortion is somehow related to biological function. Here we further analyze previous data and perform some new strategic single-molecule and computational experiments in order to assess the possibility that $\Sigma$-DNA may have fundamental roles in biology.

Our hypothesis is that $\Sigma$-DNA is an inhomogeneous structure consisting of stacked triplets of nucleobases, with base planes arranged perpendicularly as in $B$-DNA, and that these triplets are separated by empty gaps. Such a triplet structure may have a biological role in enhancing the recognition of complementary base sequence and promote the strand exchange process in gene recombination. We further speculate that the base triplets separated by gaps may be a physical origin of the occurrence of three letters in the genetic code.

\section{Materials and methods}

See online SI (Sections 1-3) for details of methods, materials and calculations. 

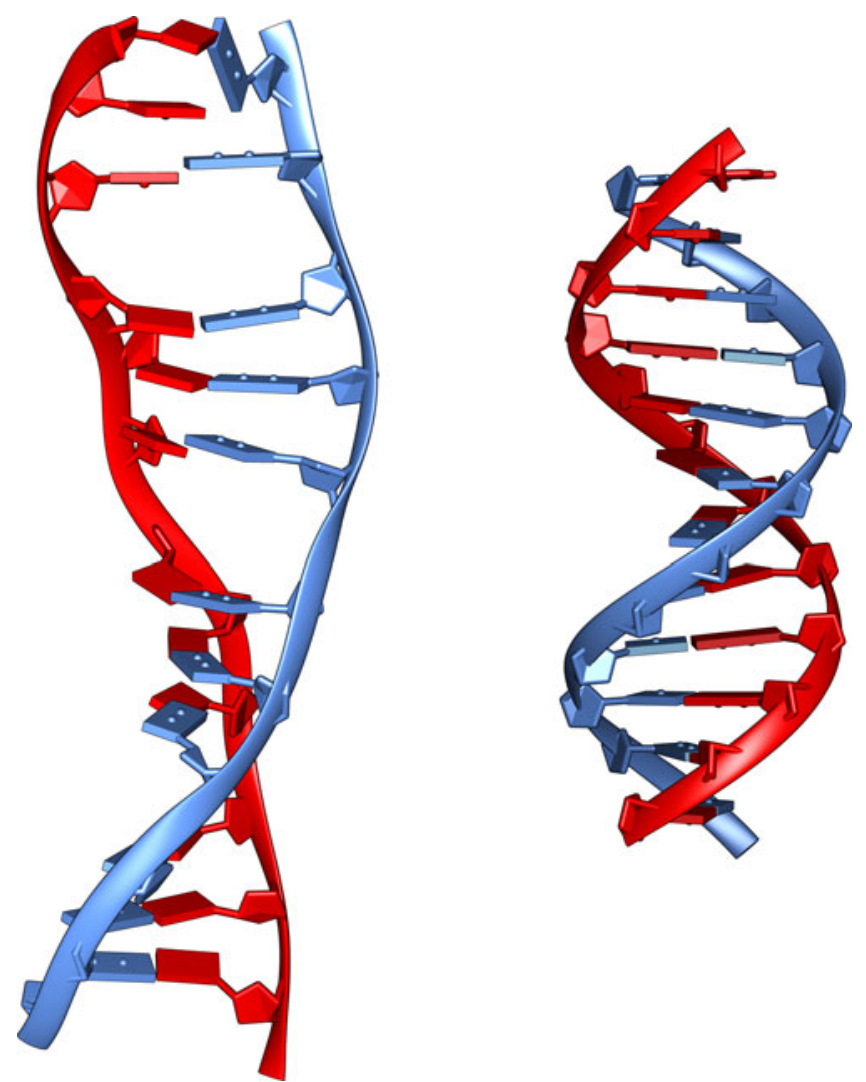

Fig. 1. The DNA structure inside a RecA-dsDNA filament according to crystal structure (left) (Chen et al. 2008) compared with freesolution double-stranded $B$-form DNA (right).

\section{Results}

Mixed-sequence long plasmid or phage dsDNA when pulled mechanically undergoes an elongation by about $70 \%$ at 60 $70 \mathrm{pN}$ (Cluzel et al. 1996; Williams et al. 2002), partly involving fuzzy denaturation. This is also what we observed with short synthetic AT-rich DNAs (Bosaeus et al. 2012, 2014). In contrast, we found more GC-rich DNAs to populate a well-defined conformation with a contour length ca $50 \%$ longer than B-form DNA (Bosaeus et al. 2012, 2014), i.e. nearly the same degree of extension that DNA has in its complex with RecA (Fig. 1).

Here, we further analyze our earlier results (Bosaeus et al. 2012) and perform additional experiments on GC-rich DNA sequences (see online SI Section 1 'DNA oligomers sequences'), in order to better characterize the transition to the $\Sigma$-DNA conformation and its structure. Critical values of force and extension at the $B \rightarrow \Sigma$ transition point, $\left(B \rightarrow S\right.$ for $5^{\prime}-5^{\prime}$ and $\left.5^{\prime}-3^{\prime}\right)$ together with free energy changes, are collected in Table 1. Figure 2 shows a typical example of response when force is applied to the 3 '-ends of a double helix, resulting in an overstretched base-paired DNA conformation, as previously reported (Bosaeus et al. 2012). By probing the response when the stretching force instead is applied to the 5 -ends, or to both ends of one strand $\left(3^{\prime}\right.$-to- $\left.5^{\prime}\right)$, we aimed to gain further insight about the conformational flexibility of DNA. However, we discovered that the $5^{\prime}-5^{\prime}$ and $5^{\prime}-3^{\prime}$ stretch transitions in our short synthetic DNA appear much less distinct and, furthermore, occur at a significantly higher transition force than the $3^{\prime}-3^{\prime}$ case (Table 1). Although RecA and Rad51 may use intrastrand interactions to stretch DNA (Xu et al. 2017), we therefore here confine ourselves to the $3^{\prime}-3^{\prime}$ case, which is associated with the distinct $\Sigma$ conformation in the absence of recombinase enzymes, and analyze this closer.

As shown in Fig. 2, stretching a single 60 bp GC-rich (60\% GC) molecule can be well described statistically in terms of a two-state thermodynamic model. As shown by the forcetrap position during pull (blue) and relaxation (red), the DNA duplex exhibits bistability at $61-65 \mathrm{pN}$, with no detectable hysteresis between pull and relax. Data (circles) pooled from 16 pull-and-relax cycles on one molecule (DNA1; for sequence, see online SI Section 1) and time trace of force in overstretch region show a distribution during time intervals of $1 \mathrm{~s}$. A different sequence but with the same total GC content (DNA2) shows within experimental error identical results indicating that the transition to $\Sigma$-form is not markedly sequence dependent. 
Table 1. The table lists the force response at room temperature in $1 \mathrm{M} \mathrm{NaCl}$ buffer of different DNA constructs based on a double stranded region containing $60 \%$ GC (see online SI Section 1 for sequences)

\begin{tabular}{|c|c|c|c|c|c|c|}
\hline Name & Geometry & No. bp & $F_{\mathrm{tr}}(\mathrm{pN})$ & $\Delta x @ F_{\mathrm{tr}}(\mathrm{nm})$ & $\Delta G\left(\mathrm{kcal} \mathrm{mol}^{-1} \mathrm{bp}^{-1}\right)$ & $N$ \\
\hline DNA1 & $3^{\prime}$-to-3' & 60 & $63 \cdot 3 \pm 1 \cdot 11$ & $10 \cdot 4 \pm 0 \cdot 42$ & $1.66 \pm 0.23$ & 49 \\
\hline $\mathrm{L}^{\prime} \mathrm{R}^{\prime}-\mathrm{DNA} 1$ & $3^{\prime}$-to-3' & 64 & $62 \cdot 5 \pm 1 \cdot 22$ & $11.4 \pm 0.75$ & $1 \cdot 61 \pm 0 \cdot 19$ & 10 \\
\hline L'-DNA1 & $3^{\prime}$-to- $3^{\prime}$ & 64 & $62 \cdot 0 \pm 1 \cdot 18$ & $11 \cdot 5 \pm 0.91$ & $1.64 \pm 0.26$ & 34 \\
\hline R'-DNA1 & $3^{\prime}$-to- $3^{\prime}$ & 64 & $63.7 \pm 1.07$ & $11.0 \pm 0.73$ & $1.52 \pm 0.28$ & 11 \\
\hline S3'3'-DNA1 & $3^{\prime}$-to-3' & 60 & $63 \cdot 5 \pm 1 \cdot 24$ & $10 \cdot 5 \pm 0.75$ & $1.50 \pm 0.27$ & 17 \\
\hline S5'5'-DNA1 & $5^{\prime}$-to- $5^{\prime}$ & 60 & $67 \cdot 5 \pm 2 \cdot 33$ & $7 \cdot 80 \pm 0.99$ & $1 \cdot 13 \pm 0.29$ & 8 \\
\hline S5'3'-DNA1 & $5^{\prime}$-to- $3^{\prime}$ & 60 & $66 \cdot 4 \pm 1.72$ & $6 \cdot 05 \pm 2 \cdot 42$ & $1 \cdot 15 \pm 0 \cdot 21$ & 10 \\
\hline DNA2 & $3^{\prime}$-to- $3^{\prime}$ & 60 & $62 \cdot 0 \pm 1 \cdot 77$ & $11 \cdot 4 \pm 0.47$ & $1 \cdot 58 \pm 0 \cdot 17$ & 33 \\
\hline$(\mathrm{DNA} 1)_{2}$ & $3^{\prime}$-to-3' & 122 & $64 \cdot 2 \pm 1 \cdot 56$ & $23 \cdot 8 \pm 1 \cdot 92$ & $1.57 \pm 0.12$ & 17 \\
\hline
\end{tabular}

Geometry - positions of the single-stranded DNA handles transmitting the force. $F_{\text {tr }}$, equilibrium transition force; $\Delta x @ F_{\text {tr }}$ molecular extension during transition measured at the transition force; $\Delta G$, free energy difference between states; $N$, number of molecules in the set.

Measurements displaying the force versus distance spectrum contain thermodynamic and kinetic information. One observable is the transition frequency when the force is tuned to the point of symmetric switching between the two conformations, corresponding to equilibrium constant $K=1$. Our observation that the frequency is relatively low (typically $100 \mathrm{~Hz}$ for $60 \mathrm{bp}$ DNA) indicates that it is not a single-base destacking scenario (expected to be ns) that we are observing but an avalanche involving a large number of bases turning coherently from one structure to another (coherence length estimated to be as large as $50 \mathrm{bp}$, see below). This is a large cooperativity, part of which may be understood in classical mechanical terms.

Figure 3 shows the force spectrum for a 122 bp DNA concatamer consisting of two identical $60 \mathrm{bp}$ GC-rich sequences connected covalently in series ((DNA1) $)_{2}$; for sequence, see online SI Section 1). The result can be understood in mechanical terms: once one of the two segments yields to force, then the slack will imply decreased force on the second half of the molecule. The transition force for the $122 \mathrm{bp}$ DNA is similar to the force observed in Fig. 2 for one 60 bp DNA (64.2 pN compared with $63.3 \mathrm{pN})$.

The system may be described in terms of three nondegenerate states as shown in Fig. $3 b$ and $3 c$. From the data in Fig. $3 c$, one may deduce the cooperative length in the same way as was performed for the result in Fig. 2 (see online SI Section 2): the cooperative length as measured is $8 \mathrm{~nm}$ for the first transition and $12 \mathrm{~nm}$ for the second transition corresponding to an average extension of 23.8 $\mathrm{nm}$ or a cooperative length of 41 base pairs (to be compared with $50 \mathrm{bp}$ for the $60 \mathrm{bp}$ DNA molecule). The exactness by which the system can be described by three thermodynamic states is remarkable (Fig. $3 c$ ). The total stretching of the DNA dimer corresponds to an extension ratio of 1.54 \pm 0.04 .

Figure 4 shows the two models of stretched DNA that we consider to be feasible, given the deformation and energetics observed: the continuous one with strongly slanted bases (to the right, called $\Sigma^{\prime}$ ) and the inhomogeneous model with stacked base triplets ( $\Sigma$, in the middle). In the SI part of (Bosaeus et al. 2012) a simple energy estimate was made to compare orthogonal and slanted base arrangements, which we here expand upon. Denoting $L$ the length of DNA helix, $N$ the number of bases, $l_{\mathrm{G}}$ the gap size and $l_{\mathrm{B}}$ the base rise, $r_{\mathrm{B}}$ the base length, and $n$ the number of clustered bases in the inhomogeneous models and $A$ and $E$ the stacking surface and energy, respectivly, one has for the case of slanted bases (right, $\theta$ base tilt angle): $L=l_{\mathrm{B}} N \cos \theta$ and $E$ (tilted) $=\mathrm{AN}[1-$ $\left.\left(2 l_{\mathrm{B}} \tan \theta\right) / r_{\mathrm{B}}\right]$. With $50 \%$ lengthening $L=1.5 l_{\mathrm{B}} N$ gives $\theta=$ $48^{\circ}$. Stacking energy for $\theta=48^{\circ}$ tilt and base length $r_{\mathrm{B}}=10$ $\AA$ and width $l_{\mathrm{B}}=3.4 \AA$ : $E_{\mathrm{T}}=0.25 \mathrm{AN}$. Correspondingly, for the orthogonal, inhomogeneous model (middle) assuming $l_{\mathrm{B}}=3.4 \AA$, one has for $50 \%$ lengthening $l_{\mathrm{G}}=5 \AA$ with $n=3$. Stacking energy $E$ (orthogonal) $=0.50 \mathrm{~A}$ for $n=2$ and 0.66 A for $n=3$.

With this crude estimate, we conclude that the inhomogeneous perpendicularly stacked DNA model with base triplets is the most stable one provided that we may assume (know) that the bases are perpendicular. A question is how much more stable is the triplet compared with quadruplet and doublet arrangements? We discard the $n=4$ case because of too high backbone strain (estimated to be $>5 \mathrm{kcal} \mathrm{mole}^{-1}$ quadruple base pair), while doublets are still conceivable: using the stacking free energy determined by Kool et al. from a single-stacked base, the energy penalties for 50\% stretched perpendicular base arrangements are: 9, 6 and $3 \mathrm{kcal}$ per base pair, respectively, for homogeneous perpendicular, doublet and triplet arrangements compared with stacked $B$-DNA. Thus, the triplet is the energetically most economical conformation. The triplet state may also be concluded to be the energetically optimal cutoff for the many-body van der Waals interactions arising between DNA bases (Distasio et al. 2012). We have then not considered stabilization due to entropic contributions which Harris et al. have found is important (Harris et al. 2005; Řezác et al. 2010; Sutthibutpong et al. 2016). Note that 
(a)

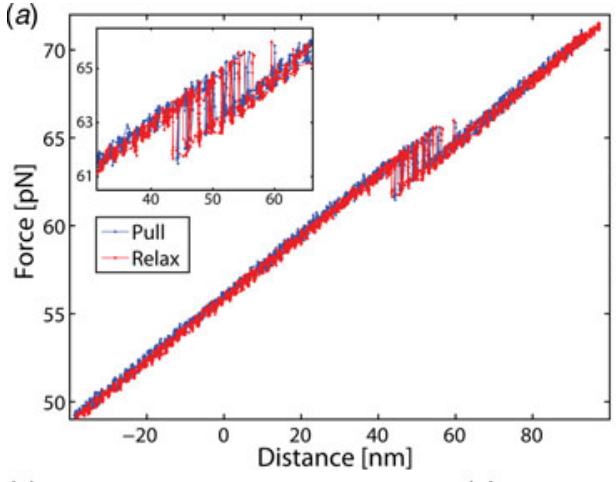

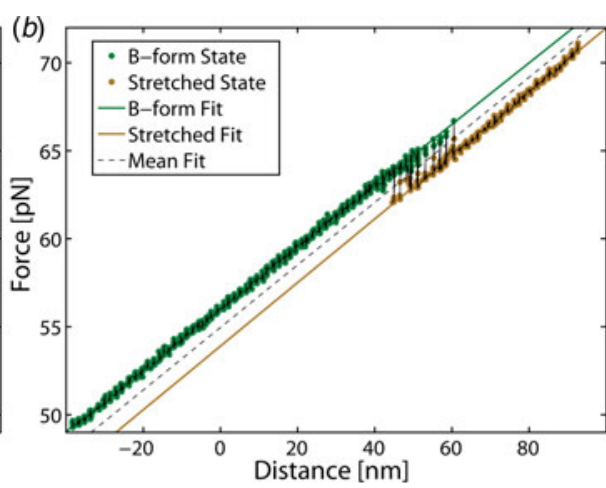

(d)

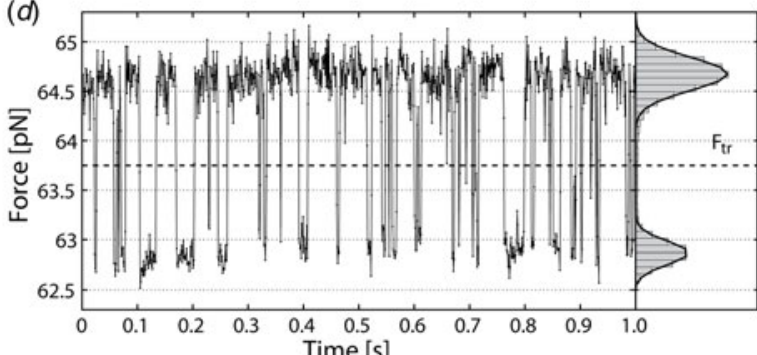

(e)

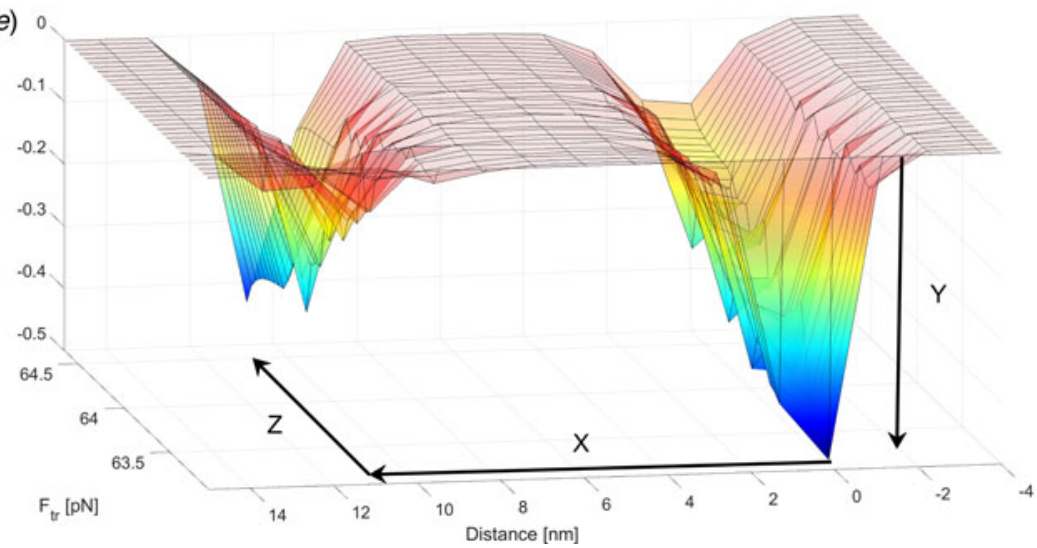

Fig. 2. Stretching a single $60 \mathrm{bp}$ GC molecule (a) force-trap position during pull (blue) and relaxation (red). DNA duplex exhibits bistability at $61-65 \mathrm{pN}$, with no detectable hysteresis between pull and relax. (b) Force position during single pulling, illustrating two states. (c) Probability of finding DNA in overstretched state as a function of force. Data (circles) pooled from 16 pull-and-relax cycles on one molecule. $(d)$ Time trace of force in overstretch region. Histogram (right) gives distribution during time interval of $1 \mathrm{~s}(a-d$ reproduced with permission from (Bosaeus et al. 2012)). At bottom (e) an energy landscape is generated based on the experimental data: $y=$ free energy stabilization in $B$-form ca $1.5 \mathrm{kcal} \mathrm{mol}^{-1}$ base pairs, $x=$ stretch displacement $11.0 \mathrm{~nm}, z=$ force ca $64 \mathrm{pN}$. Elongation: from $21.4 \mathrm{~nm}(B$-DNA) to $32.4 \mathrm{~nm}(\Sigma$-DNA), i.e. elongation factor $1.51 \pm 0.05$. The corresponding average elongation with double DNA (122 base pairs) was 1.54 .

both experiments and steered molecular dynamics (MD) simulations suggest that $S$-DNA is a strongly extended structure with slanted bases (average $\theta<50^{\circ}$ ) prone to form large denaturation bubbles - in agreement with our observations of the behaviour of AT-rich DNA (where covalent glyoxal probing confirms denaturation) (Bosaeus et al. 2012, 2014). This variation emphasizes the difference between earlier $S$-forms and our new $\Sigma$-DNA conformation. However, accurate quantification of various energy terms in DNA base pairing is still a challenge. Even experiments addressing DNA base stacking have large variations: for instance, values for AA stacking can be between $-1.2 \mathrm{kcal}$ $\mathrm{mol}^{-1}$ (Mitchell \& Sigel, 1978) and $-5.73 \mathrm{kcal} \mathrm{mol}^{-1}$ (Morcillo et al. 1987), depending on the applied conditions. To address energy components, base stacking was studied earlier both by MD simulations as well as quantum mechanical (QM) calculations (Friedman \& Honig, 1995; Giudice et al. 2003; Kabeláč et al. 2005; Řezáč \& Hobza, 2007; Swart et al. 2007). As summarized in online Table S1, hydrogen bonds formed between the base pairs have a large contribution to base stability, however, accompanied with a large hydration-free energy per base, which may contribute to destabilization of Watson-Crick (WC) hydrogen bonds. Entropy terms based on QM normal mode analysis 

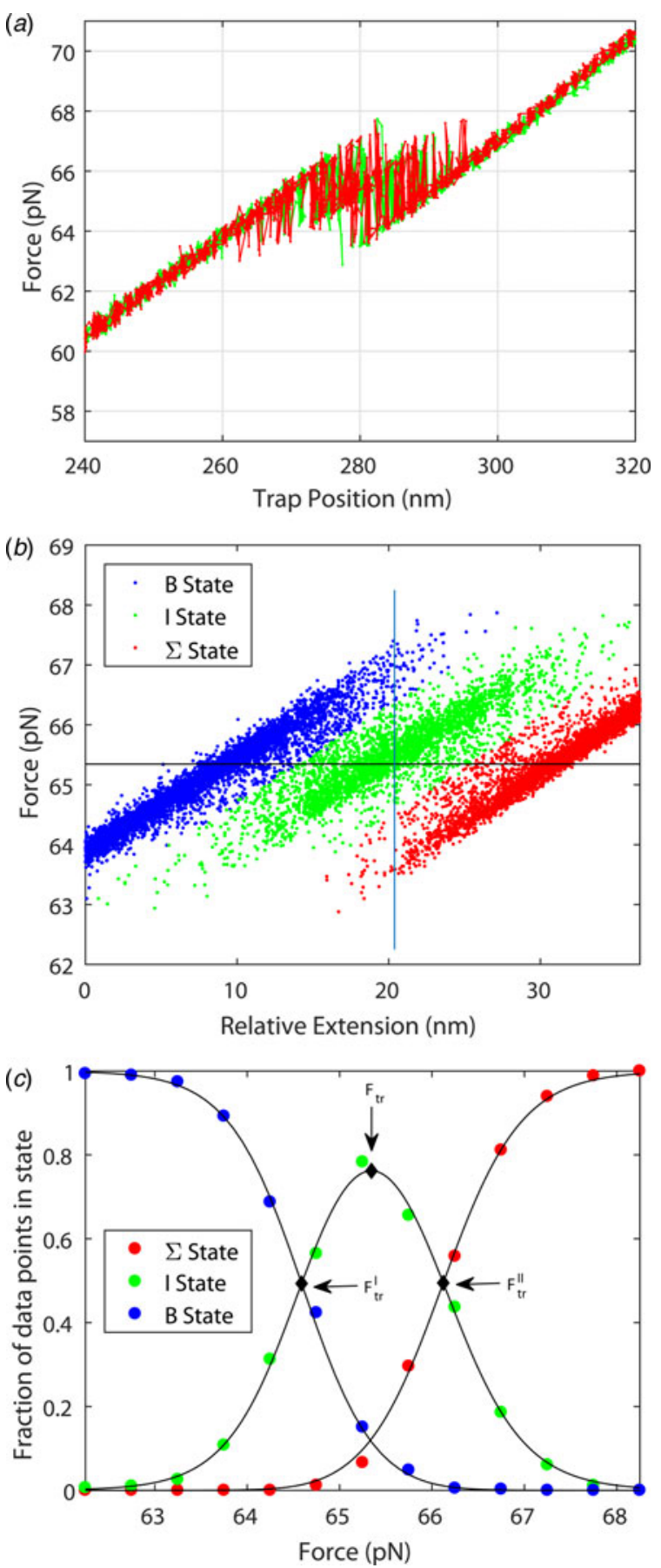

Fig. 3. Stretching a double $60 \mathrm{bp}$ GC-rich DNA molecule $\left((\mathrm{DNA1})_{2}, 122 \mathrm{bp}\right.$, for sequence see online SI Section 1). (a) Multiple pull/relax cycles on a dimer in $1 \mathrm{M} \mathrm{NaCl}$. The results show that the DNA-dimer overstretches at about the same force as the DNA monomer and that the extension is twice that of the monomer. $F=64.2 \pm 1.56 \mathrm{pN}, \Delta x=23.8 \pm 1.92 \mathrm{~nm}$ (L'DNA1: 62.0 $+1.18 \mathrm{pN}, 11.5 \pm 0.91 \mathrm{~nm}$ ). (b) For analysis purposes, each measured set of data points during the pull/relax cycles of the molecule is assigned to one of three states based on the proximity to linear fits describing each state. (c) Fractional occupancy of the states as a function of applied force; solid lines show the fitted non-degenerate three-state model (see online SI Section 2 'Fitting a three state model'). are also significant, further decreasing the large stabilization by hydrogen bonds. The non-polar contribution from base stacking was estimated to be similar by several techniques, in the range of $\simeq 5-9 \mathrm{kcal} \mathrm{mol}^{-1}$ (Friedman \& Honig, 1995; Kabeláč et al. 2005). Due to difficulties to obtain remaining entropy and hydration terms as well as nonadditive parts of stacking contributions, accurate energetic details remain obscure.

From a more general perspective, the energetic problem is challenged due to the non-symmetric nature of AT and GC base pairs. In online Table S2, we report data for calculations of stacking stabilization using QM computations for a planar benzoic-acid hydrogen-bonded dimer, which we view as a primitive symmetric model for a DNA base pair. We have also considered effects of surrounding environment on hydrogen bond strength, an aspect that previous gas phase QM calculations on DNA base pairs did not address. Our results confirm that the hydrogen bonds are significantly strengthened by a non-polar matrix. We investigated typical pairwise interactions identified between DNA base pairs (Kabeláč et al. 2005), i.e. WC-type H-bonded (WC), intramolecular stacking (S), and interstrand stacking (IS) (online Fig. S1). The WC $\mathrm{H}$-bonds are partially destabilized and $3.0 \mathrm{kcal} \mathrm{mol}^{-1}$ weaker in water than in an apolar, e.g. cyclohexane, environment (online Table S2). In contrast, for intrastrand stacking, S, the stacking of base pairs is more favoured in water by $1.2 \mathrm{kcal}$ $\mathrm{mol}^{-1}$, while interstrand stacking, IS, is nearly identical in aqueous and apolar matrices $\left(\Delta \Delta E=-0.12 \mathrm{kcal} \mathrm{mol}^{-1}\right)$. These results are in line with the increased strength of buried H-bonds (Deechongkit et al. 2004) and also known from the experimental appearance of strong hydrogen-bonded dimers in polyethylene compared with water solution (Norden, 1977).

Further strengthening of base-pair stacking is due to contribution from dispersive forces as shown by $\pi$-stacking of two benzoic-acid dimers (online Fig. S1). Similar conclusions are found in the literature (Da̧bkowska et al. 2005; Friedman \& Honig, 1995). The magnitude and nature of the individual interactions are similar to previous high-level QM calculations performed on DNA base pairs (Kabeláč et al. 2005; Swart et al. 2007) and also on benzene dimers (Swart et al. 2007). In the latter study, benzene dimers were also considered and coupled cluster with single double and perturbative triple excitation $[\operatorname{CCSD}(\mathrm{T})]$ and Keal \& Tozer functional (KT1) calculations resulted in stacking energies close to those determined here for the benzoic acid dimers (for more details, see online SI Section 3 'Calculations of DNA stacking energies'). A great advantage of the benzoic acid model is its symmetric nature, where we may also gain insight into relative energy gains of adding an additional stacked dimer into the construct without considering the base-pair variance arising from the asymmetry of the four bases plus their sequential ordering. The obtained energy values for adding on dimers demonstrate, similar to a many-body van der Waals stacking interaction (Distasio et al. 


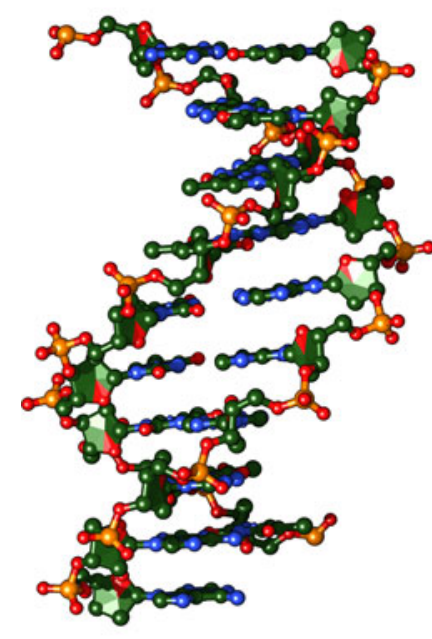

B

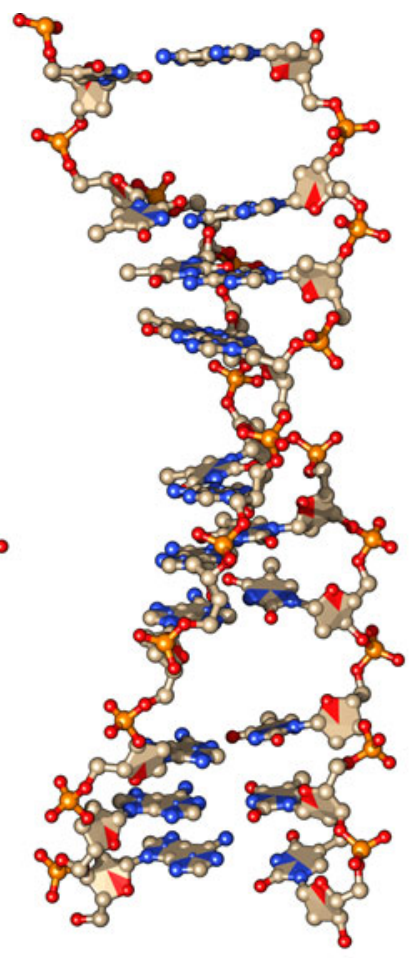

$\Sigma$

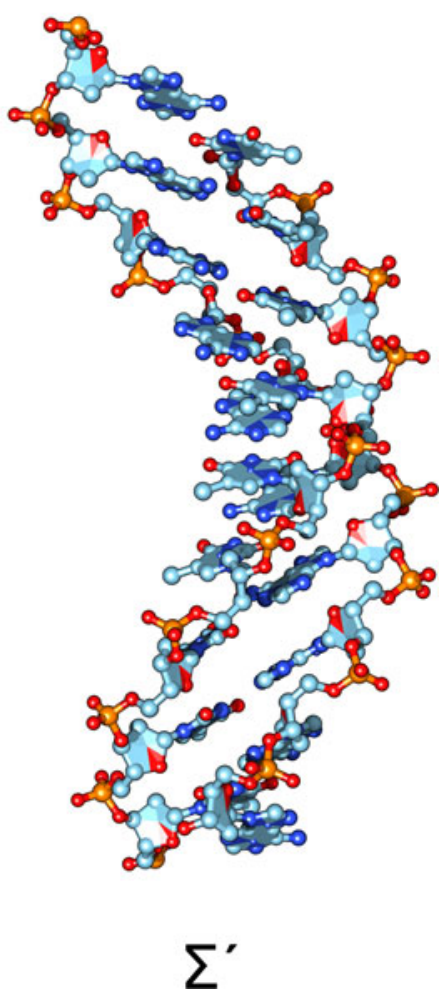

Fig. 4. Two energetically plausible stretched $\Sigma$-DNA structures compared with $B$-DNA (left): inhomogeneous disproportionation triplet form with perpendicular bases (middle, $\theta=75-90^{\circ}$ ), homogeneous form with inclined bases (right, $\theta=48^{\circ}$ ).

2012), that the relative change in energy gain per benzoic acid dimers becomes significantly reduced after the third base pair is added (online Fig. S2). The energy gain upon adding a third benzoic acid pair to a dimer of pairs is nearly double that for adding a fourth pair to a trimer of pairs or a fifth pair to tetramer of benzoic acid pairs (for details, see online SI Section 3).

Regarding the disproportionation mechanism, it appears that the relatively small force $(60-70 \mathrm{pN})$ will allow various stacked regions of an extended DNA, to compete with each other. Then mainly stochastic effects will determine which stacked conformation will break up or lose one stacked pair from the end of a $B$-DNA fragment. Considering a hypothetical-stacked benzoic acid filament, when the pulling force makes a triplet and a quadruplet compete, then changing to a doublet from a triplet would mean a loss of $2.95 \mathrm{kcal}$ $\mathrm{mol}^{-1}$ stabilization energy, compared with changing from a quadruplet to triplet, which costs a mere $1.45 \mathrm{kcal} \mathrm{mol}^{-1}$. This suggests that in case of DNA, the smaller force applied during pulling will probably render all longer stacked regions to break up, except for those that are in triplet form, where the split into a doublet will be too energy demanding. Again, this supports the formation of $\Sigma$-DNA, with triplets, to occur.

The transition force we obtained for $\Sigma$ conformation ( $64 \mathrm{pN}$ ) corresponds to a free-energy change of ca $1.5 \mathrm{kcal}$ per mol base pair, which compares fairly well with the experimental end-stacking energy of 2-6 kcal mol${ }^{-1}$ for an AT base pair (Guckian et al. 2000; Kool, 2001). Note that our energy diagram (Fig. 2) does not include any activation barrier for the transition from $B$ to $\Sigma$ forms. An accurate determination of the activation energy will require precise kinetic data at varied temperatures.

\section{Discussion}

Laser-tweezers force-spectroscopic data (Bosaeus et al. 2012, 2014) on short DNA of variable sequence are here used as basis for discussing the structural and energetic properties of $\Sigma$-DNA and to put forward a disproportionation hypothesis.

The study of DNA-stretching mechanisms could give us several important clues about DNA physical properties in general and for the homology search of genetic recombination in particular. It is yet to be determined which conformation of pure stretched DNA is more stable: an inhomogeneous conformation $(\Sigma)$ with perpendicular bases, or a homogeneously stacked structure with slanted bases $\left(\Sigma^{\prime}\right)$. Our energetic arguments are in favour of the inhomogeneous (disproportionated triplet gap) $\Sigma$-form, but this rests on the ad hoc assumption of perpendicular bases for which there is as yet no structural evidence, except for in DNA complexes with 
RecA (Fig. 1). Nonetheless, the observations of an energetically feasible transition force (consistent with base-pair triplet stacks) as well as the same extension length as observed for DNA in complex with RecA (Fig. 1) strongly argue that the $\Sigma$-form of DNA is a metastable structure that can form when alone free in solution.

Because the conformation of stretched DNA may be identical to that promoted by RecA, we would like to speculate about the possible role of DNA stretching and triplet arrangement in RecA-promoted genetic recombination. As suggested by Taghavi and Berryman and supported by their detailed modelling (under consideration in QRB Discovery), the presence of some intercalating cationic ligand could provide an energetic bias favouring perpendicularity and the triplet kind of disproportionation in DNA. In our LD-based model for the Rad51-dsDNA structure, we suggested one tyrosine to be intercalated as its orientation would be consistent with such a structure (Reymer et al. 2009), and this is clearly an attractive mechanism to explain the triple base stack. However, full intercalation might not be a necessary condition for disproportionation to occur, as MD simulations of Rad51-DNA complexes have shown that although a positive-charge moiety (tyrosine) is positioned close to the gap between the two base triplets in the starting structure, the evolving structure stays essentially stable (over $10 \mathrm{~ns}$ ) and the tyrosine does not enter the potential intercalation slot (A. Reymer, unpublished data). For both RecA and Rad51, the presence of ATP (or non-hydrolyzable ATP $\gamma$ S) is needed for the formation of the extended recombinase-DNA complexes, both for ssDNA as well as for dsDNA, although with ssDNA a different, unstructured complex can form in absence of ATP (Williams \& Spengler, 1986). Although ATP is a putative candidate for intercalation, both LD and fluorescence data (for etheno-adenine) indicated that the adenine plane of ATP was not intercalated (Takahashi \& Nordén, 1994) and this was also verified in the later X-ray structure (Chen et al. 2008).

We propose that base triplets might serve as recognition elements in the search for homology step of RecA and that physical properties of nucleic acids favouring triplet grouping relate to the triplets of codon/anticodon RNA. It has been proposed, based on dual-molecule manipulation studies, that the distance between the DNA-binding sites governs the fidelity of homology recognition (De Vlaminck et al. 2012). An extended, yet locally dsDNA conformation seems to match the way both ssDNA and dsDNA are distorted and line up in parallel inside the RecA filament. The base-pair matching in the case of a stacked triplet package can be anticipated to be more critical and exact than if the bases were moving freely. This is an alignment effect like for the sections of a key which are rigidly oriented perpendicular to the shaft of the key to fit critically in the lock. The unstacking of $B$ form into $\Sigma$ form will provide some destabilization, but this may be balanced by this local orientational recognition stability. Furthermore, the newly formed duplex DNA inside the RecA tube, after strand exchange, will then also be in $\Sigma$ form. This means in turn that it is less stable and presumably more sensitive to sequence heterogeneity (mismatch), which may permit quick discrimination and, in absence of acceptable complementarity, promote back-reaction and dissociation. In accord, for homologous DNA, the complex of dsDNA and RecA/ssDNA has a half-life of seconds (Xiao et al. 2006) and for non-homologous DNA even shorter. Molecular modelling suggests that $B$-DNA is deformed towards a curved form with kinks appearing during homology search by RecA filament (Saladin et al. 2010). Distortion of dsDNA has been observed also in joint complexes of RAD51/ssDNA by scanning force microscopy (Ristic et al. 2011).

Our new experiments show that application of force to opposite strands $\left(3^{\prime}-3^{\prime}\right.$ or $\left.5^{\prime}-5^{\prime}\right)$ of dsDNA differs from when the force is applied to the same strand $\left(3^{\prime}-5^{\prime}\right)$ (see Table 1). Quite surprisingly, the $3^{\prime}-3^{\prime}$ case also differs significantly from $5^{\prime}-5^{\prime}$, implying that the structure feels the diastereomeric effect of the ribophosphate chirality and the helical winding of the double helix. Such results (also observed by others (Danilowicz et al. 2009)) provide information about interactions of the distorted nucleic acid conformations, and may hint about a broken symmetry, so that the first and third base of a triplet stack would differ from each other. Note that the observation of insignificant differences in transition properties between $5 \mathrm{mM}$ and $1 \mathrm{M} \mathrm{NaCl}$ is in agreement with the concept of highly stable stacked triplets surrounded by a high concentration of counterions regardless of added $\mathrm{NaCl}$. The diastereomeric effect should be further analyzed but is beyond the scope of this study.

The high quality of the force-distance data and their nearly perfect quantitative fit to the two-state thermodynamic model for the 60-mer DNA are remarkable observations that demonstrate both accuracy of the experimental setting as well as that the conformational transition is well defined. For example, if the transition path had involved significant amounts of long-lived bulges or other trapped conformational species, they would have been revealed as deviations in the oscillatory read-out (Fig. 2d). The good fit for the double sequence is also remarkable (Fig. 3), populating the three combinatorial thermodynamic states almost as quantitatively predicted. The high cooperative length $(50 \mathrm{bp})$ for the $60 \mathrm{bp}$ dsDNA is another indication of a well-defined global conformation without any significant, sequence-dependent local conformations. The slightly shorter cooperative length for the double-sequence DNA (41 bp) might reflect a kinetic imperfectness in the way thermal fluctuations populate the three combinatorial states, or is an entropic effect.

No connection between triplet base conformation and origin of the genetic code has been proposed before, as far as we know, while RNA templating of amino acids has been 
speculated as a possible code origin in an RNA-world context (Koonin \& Novozhilov, 2009; Yarus et al. 2010). In support of our hypothesis of stacked base triplets as intrinsic recognition elements based on physical properties, we note that base-stacking arrangements also appear in mRNA/ribosome/tRNA complexes (Selmer et al. 2006). Along the line of our speculation about a physical (conformational) origin of the genetic code, together with our observation of a broken symmetry for single-molecule $3^{\prime}-3^{\prime}$ and $5^{\prime}-5^{\prime}$ stretch experiments, the polarity of triplet base-pair stacks could be discussed. Already Crick postulated the 'Wobble' hypothesis to account for the fact that most organisms have $<64$ (often fewer than 45) species of tRNA: the $5^{\prime}$ base on the anticodon (which binds to the $3^{\prime}$ base of mRNA) is not as spatially confined as the other two bases (Crick, 1966) As an example, yeast tRNA(Phe) has the anticodon 5'-GmAA-3' and can recognize the codons 5'-UUC-3' and 5'-UUU-3'. The two-out-of-three hypothesis of Lagerkvist (Lagerkvist, 1978) explains the synonyms and degeneracy of the code in terms of structural (possibly chemical) ambiguity of the third base.

The origin of the genetic code is part of the question of origin of life (Lagerkvist, 1978) and, of course, then any progress could be disruptively important. However, here we confine ourselves to the role of base stacking, which is the most important stabilizing energy term in DNA doublehelix motifs (Kool, 2001), and if recognition of triplet base aggregates by hydrogen bonding and steric effects may be responsible for high-fidelity self-recognition mechanisms, in RecA and generally. Returning to the question how RecA exploits the triplet DNA base stack, there are no indications of any preferred sequence of three bases, but the triplets are stochastically composed along the genetic sequence in complex with RecA. RecA will guarantee sequence neutrality by not interacting significantly with the nucleobases but binding only to the phosphate backbone. Still the stacking will contribute to stability, so that any mechanism testing homology will benefit from the freedom of dealing with each triple-base stack as a discrete unit, and at the same time have decreased base-pairing stability.

In conclusion, despite the importance of recombinases in many contexts (e.g. human diseases and treatments) and many years' intense research, their mechanistic functions in performing search for homology and executing strand exchange are not yet understood at an atomic level. Thus, while nucleobase recognition, in replication and translation contexts, has been studied extensively and is mechanistically relatively well understood, much remains to unveil about recombination reactions in general and the role of extension of DNA in particular. Here the knowledge from singlemolecule force spectroscopy could add importantly to our mechanistic understanding. Thermodynamic studies confirm that with both DNA and RNA, the stabilizing free energy is dominated by hydrophobic and dispersive nucleobase-stack interactions, while base-pair hydrogen bonding, electrostatic and stereochemical effects, crucial for recognition fidelity, are less prominent or even repulsive (Kool, 2001). Our results show that the $\Sigma$ conformation in GC-rich DNA is characterized by a clear two-state transition with a substantial cooperative length, emphasizing a model where a disproportionated triplet base structure may have a leading role in recombinase function and possibly also in other nucleic acid recognition contexts.

\section{Speculation box}

We speculate that the base triplets constituting the genetic code may have their origin in the physical preference of triplet base stacking in stretched nucleic acids, represented by the $\Sigma$ conformation of DNA. Such a metastable but distinct conformation may improve discrimination between match and mismatch base pairing in homology search reactions, present already in the earliest organisms.

\section{Supplementary material}

The supplementary material for this article can be found at https://doi.org/10.1017/S0033583517000099.

\section{Acknowledgements}

The authors acknowledge the Swedish Research Council (AR, BN and PW-S) and the Wallenberg foundation (PWS) for financial support. The support for TB-S by the Hungarian Momentum programme (LP2016-2), by the National Competitiveness and Excellence Program (NVKP_16-1-2016-0007) and by the BIONANO_GINOP$2 \cdot 3 \cdot 2-15-2016-00017$ project is greatly appreciated.

\section{Conflict of interest}

None.

\section{References}

Arnott, S., Chandrasekaran, R., Millane, R. P. \& Park, H. S. (1986). DNA-RNA hybrid secondary structures. Journal of Molecular Biology 188, 631-640.

Bosaeus, N., El-Sagheer, A. H., Brown, T., Smith, S. B., Akerman, B., Bustamante, C. \& Norden, B. (2012). Tension induces a basepaired overstretched DNA conformation. Proceedings of the National Academy of Sciences of the United States of America 109, 15179-15184.

Bosaeus, N., El-Sagheer, A. H., Brown, T., Åkerman, B. \& Nordén, B. (2014). Force-induced melting of DNA - Evidence for peeling and internal melting from force spectra on short synthetic duplex sequences. Nucleic Acids Research 42, 8083-8091. 
Brown, B. A., Lowenhaupt, K., Wilbert, C. M., Hanlon, E. B. \& Rich, A. (2000). The zalpha domain of the editing enzyme dsRNA adenosine deaminase binds left-handed Z-RNA as well as Z-DNA. Proceedings of the National Academy of Sciences of the United States of America 97, 13532-13536.

Chen, Z., YANG, H. \& Pavletich, N. P. (2008). Mechanism of homologous recombination from the RecA-ssDNA/dsDNA structures. Nature 453, 489-494.

Cluzel, P., Lebrun, A., Heller, C., Lavery, R., Viovy, J.-L., Chatenay, D. \& CARON, F. (1996). DNA: an extensible molecule. Science 271, 792-794.

Crick, F.H.H. (1966). Codon-anticodon pairing: the wobble hypothesis. Journal of Molecular Biology 19, 548-555.

Dą̧BKowsKa, I., Gonzalez, H. V., JurečKa, P. \& Hobza, P. (2005). Stabilization energies of the hydrogen-bonded and stacked structures of nucleic acid base pairs in the crystal geometries of CG, AT, and AC DNA steps and in the NMR geometry of the $5^{\prime}$-d (GCGAAGC)-3' hairpin: complete basis set calculations at the MP2 and. Journal of Physical Chemistry A 109, 1131-1136.

Danilowicz, C., Limouse, C., Hatch, K., Conover, A., Coljee, V. W., Kleckner, N. \& Prentiss, M. (2009). The structure of DNA overstretched from the $5^{\prime} 5^{\prime}$ ends differs from the structure of DNA overstretched from the $3^{\prime} 3^{\prime}$ ends. Proceedings of the National Academy of Sciences of the United States of America 106, 13196-13201.

Deechongkit, S., Nguyen, H., Powers, E. T., Dawson, P. E., Gruebele, M. \& KelLY, J. W. (2004). Context-dependent contributions of backbone hydrogen bonding to beta-sheet folding energetics. Nature 430, 101-105.

De Vlaminck, I., van Loenhout, M. T. J.T. J., Zweifel, L., den Blanken, J., Hooning, K., Hage, S., Kerssemakers, J. \& Dekker, C. (2012). Mechanism of homology recognition in DNA recombination from dual-molecule experiments. Molecular Cell 46, 616-624.

Distasio, R. A., von Lilienfeld, O. A. \& Tkatchenko, A. (2012). Collective many-body van der Waals interactions in molecular systems. Proceedings of the National Academy of Sciences of the United States of America 109, 14791-14795.

Flory, J., Tsang, S. S. \& MuniYaPpa, K. (1984). Isolation and visualization of active presynaptic filaments of recA protein and singlestranded DNA. Proceedings of the National Academy of Sciences of the United States of America 81, 7026-7030.

Fornander, L. H. H., Renodon-Corniére, A., Kuwabara, N., Ito, K., Tsutsui, Y., Shimizu, T., Iwasaki, H., Nordén, B., Takahashi, M. (2014). Swi5-Sfr1 protein stimulates Rad51-mediated DNA strand exchange reaction through organization of DNA bases in the presynaptic filament. Nucleic Acids Research 42, 2358-2365.

Friedman, R. A. \& Honig, B. (1995). A free energy analysis of nucleic acid base stacking in aqueous solution. Biophysical Journal 69, 1528-1535.

Giudice, E., Várnai, P. \& Lavery, R. (2003). Base pair opening within B-DNA: free energy pathways for GC and AT pairs from umbrella sampling simulations. Nucleic Acids Research 31, 1434-1443.

GuCKIAN, K. M., KrUGH, T. R. \& Kool, E. T. (2000). Solution structure of a nonpolar, non-hydrogen-bonded base pair surrogate in DNA. Journal of the American Chemical Society 122, 68416847.
Hagmar, P., Norden, B., Baty, D., Chartier, M., Takahashi, M., Nordén, B., Baty, D., Chartier, M. \& Takahashi, M. (1992). Structure of DNA-RecA complexes studied by residue differential linear dichroism and fluorescence spectroscopy for a genetically engineered RecA protein. Journal of Molecular Biology 226, 1193-1205.

Harris, S. A. A., Sands, Z.A.A. \& Laughton, C. A. A. (2005). Molecular dynamics simulations of duplex stretching reveal the importance of entropy in determining the biomechanical properties of DNA. Biophysical Journal 88, 1684-1691.

Kabeláč, M., Zendlová, L., Řeha, D. \& Hobza, P. (2005). Potential energy surfaces of an adenine-thymine base pair and its methylated analogue in the presence of one and two water molecules: molecular mechanics and correlated Ab initio study. Journal of Physical Chemistry B 109, 12206-12213.

KiIANitsa, K. \& Stasiak, A. (1997). Helical repeat of DNA in the region of homologous pairing. Proceedings of the National Academy of Sciences of the USA 94, 7837-7840.

KooL, E. T. T. (2001). Hydrogen bonding, base stacking, and steric effects in DNA replication. Annual Review of Biophysics and Biomolecular Structure 30, 1-22.

Koonin, E. V. V. \& Novozhilov, A. S. S. (2009). Origin and evolution of the genetic code: the universal enigma. IUBMB Life $\mathbf{6 1}$, 99-111.

LAgerkvist, U. (1978). 'Two out of three': an alternative method for codon reading. Proceedings of the National Academy of Sciences of the United States of America 75, 1759-1762.

Lu, X.-J.X. J., Shakked, Z. \& Olson, W. K. W. K. (2000). A-form conformational motifs in ligand-bound DNA structures. Journal of Molecular Biology 300, 819-840.

Mitchell, P.R. \& Sigel, H. (1978). A proton nuclearmagnetic-resonance study of self-stacking in purine and pyrimidine nucleosides and nucleotides. European Journal of Biochemistry 88, 149-154.

Morcillo, J., Gallego, E. \& Peral, F. (1987). A critical study of the application of ultraviolet spectroscopy to the self-association of adenine, adenosine and 5'-AMP in aqueous solution. Journal of Molecular Structure 157, 353-369.

Morimatsu, K., Takahashi, M. \& Nordén, B. (2002). Arrangement of RecA protein in its active filament determined by polarized-light spectroscopy. Proceedings of the National Academy of Sciences of the United States of America 99, 11688-11693.

Norden, B. (1977). General aspects on linear dichroism spectroscopy and its application. Spectroscopy Letters 10, 381-400.

Nordén, B., Elvingson, C., Kubista, M., Sjöberg, B., Ryberg, H., Ryberg, M., Mortensen, K. \& Takahashi, M. (1992). Structure of RecA-DNA complexes studied by combination of linear dichroism and small-angle neutron scattering measurements on flow-oriented samples. Journal of Molecular Biology 226, 1175-1191.

Nordén, B., Wittung-Stafshede, P., Ellouze, C., Kim, H.-K., Mortensen, K. \& TAKahashi, M. (1998). Base orientation of second DNA in RecA.DNA filaments: analysis by combination of linear dichroism and small angle neutron scattering in floworiented solution. Journal of Biological Chemistry 273, 1568215686.

Reymer, A., Frykholm, K., Morimatsu, K., Takahashi, M. \& Nordén, B. (2009). Structure of human Rad51 protein filament from molecular modeling and site-specific linear dichroism 
spectroscopy. Proceedings of the National Academy of Sciences 106, 13248-13253.

ŘEZÁČ, J. \& HobZA, P. (2007). On the nature of DNA-duplex stability. Chemistry - A European Journal 13, 2983-2989.

ŘezÁ, J., HobZa, P. \& Harris, S. A. (2010). Stretched DNA investigated using molecular-dynamics and quantum-mechanical calculations. Biophysical Journal 98, 101-110.

Rich, A., Nordheim, A. \& Wang, A. (1984). The chemistry and biology of left-handed Z-DNA. Annual Review of Biochemistry 53, 791-846.

Ristic, D., KanaAr, R. \& Wyman, C. (2011). Visualizing RAD51mediated joint molecules: implications for recombination mechanism and the effect of sequence heterology. Nucleic Acids Research 39, 155-167.

Saladin, A., Amourda, C., Poulain, P., Férey, N., Baaden, M., Zacharias, M., Delalande, O., Prévost, C., Nicolas, F. \& Pr, C. (2010). Modeling the early stage of DNA sequence recognition within RecA nucleoprotein filaments. Nucleic Acids Research 38, 6313-6323.

Selmer, M., Dunham, C. M. M., Murphy, F. V.V. th, Weixlbaumer, A., Petry, S., Kelley, A. C. C., Weir, J. R. R. \& Ramakrishnan, V. (2006). Structure of the $70 \mathrm{~S}$ ribosome complexed with mRNA and tRNA. Science 313, 1935-1942.

Stasiak, A. \& Di Capua, E. (1982). The helicity of DNA in complexes with recA protein. Nature 299, 185-186.

Stasiak, A., Di Capua, E. \& Koller, T. (1981). Elongation of duplex DNA by recA protein. Journal of Molecular Biology 151, 557-564.

Story, R. M. M., Weber, I. T. T. \& Steitz, T. A. T. A. A. (1992). The structure of the E. coli recA protein monomer and polymer. Nature 355, 318-325.
Sutthibutpong, T., Matek, C., Benham, C., Slade, G. G. G., Noy, A., Laughton, C., Doye, J. P. K., Louis, A. A. A., Harris, S. A. A., JP, K. D., Louis, A. A. A. \& HARRIs, S. A. A. (2016). Long-range correlations in the mechanics of small DNA circles under topological stress revealed by multi-scale simulation. Nucleic Acids Research 44, 9121-9130.

Swart, M., van der Wist, T., Fonseca Guerra, C. \& Bickelhaupt, F. M. (2007). $\pi-\pi$ stacking tackled with density functional theory. Journal of Molecular Modeling 13, 1245-1257.

TAKAHASHI, M. \& NoRdÉN, B. (1994). The cofactor ATP in DNA-RecA complexes is not intercalated between DNA bases. Journal of Molecular Recognition 7, 221-226.

Williams, M. C., Rouzina, I. \& Bloomfield, V. A. (2002). Thermodynamics of DNA interactions from single molecule stretching experiments. Accounts of Chemical Research 35 , 159-166.

Williams, R. C. \& Spengler, S. J. (1986). Fibers of RecA protein and complexes of RecA protein and single-stranded phi X174 DNA as visualized by negative-stain electron microscopy. Journal of Molecular Biology 187, 109-118.

Xiao, J., Lee, A. M. M. \& Singleton, S. F. F. (2006). Construction and evaluation of a kinetic scheme for RecA-mediated DNA strand exchange. Biopolymers 81, 473-496.

Xu, J., ZhaO, L., Xu, Y., ZhaO, W., Sung, P. \& Wang, H.-W. (2017). Cryo-EM structures of human RAD51 recombinase filaments during catalysis of DNA-strand exchange. Nature Structural \& Molecular Biology 24, 40-46.

Yarus, M., Chen, I. A., Yarus, M. \& Harris, T. (2010). Life from an RNA world: the ancestor within. Nature 330, 758. 